Trinity University

Digital Commons @ Trinity

Psychology Faculty Research

Psychology Department

$11-2014$

\title{
Moderators and Predictors of Response to Eating Disorder Risk Factor Reduction Programs in Collegiate Female Athletes
}

T M. Stewart

Maribel Plasencia

Trinity University

H Han

$\mathrm{H}$ Jackson

Trinity University

Carolyn Becker

Trinity University, cbecker@trinity.edu

Follow this and additional works at: https://digitalcommons.trinity.edu/psych_faculty

Part of the Psychology Commons

Publication Details

Psychology of Sport and Exercise

\section{Repository Citation}

Stewart, T.M., Plasencia, M., Han, H., Jackson, H., \& Becker, C.B. (2014). Moderators and predictors of response to eating disorder risk factor reduction programs in collegiate female athletes. Psychology of Sport and Exercise, 15(6), 713-720. doi: 10.1016/j.psychsport.2014.02.006

This Article is brought to you for free and open access by the Psychology Department at Digital Commons @ Trinity. It has been accepted for inclusion in Psychology Faculty Research by an authorized administrator of Digital Commons@ Trinity. For more information, please contact jcostanz@trinity.edu. 


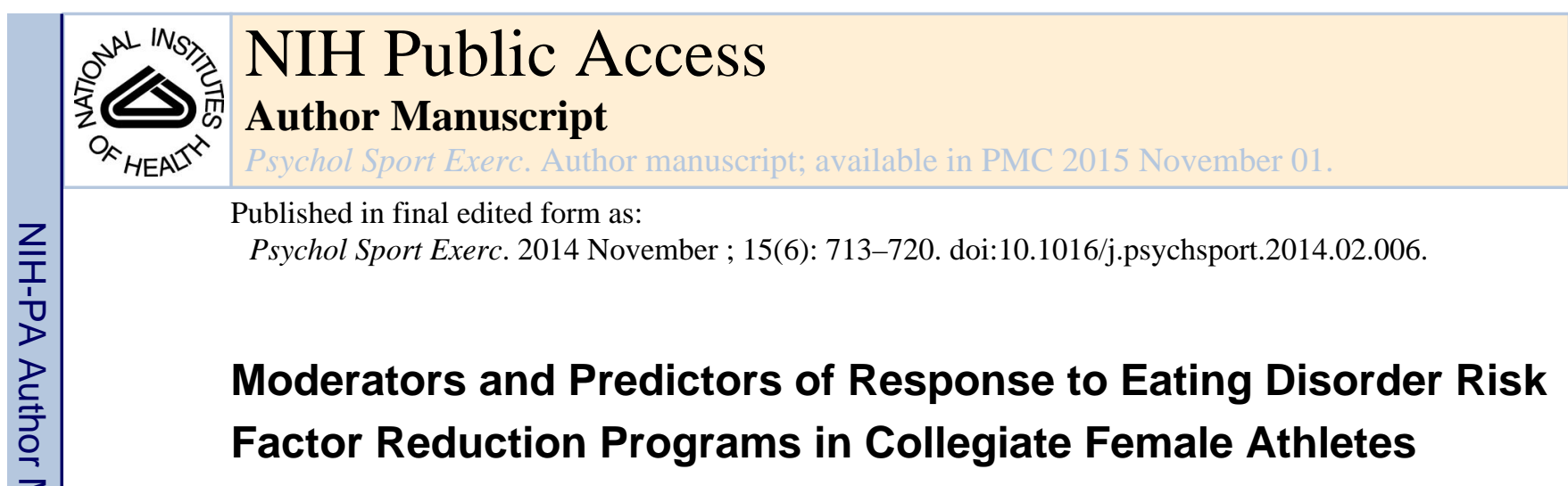

\author{
T.M. Stewart ${ }^{\mathrm{a}}$, M. Plasencia ${ }^{\mathrm{b}}$, H. Han ${ }^{\mathrm{a}}$, H. Jackson ${ }^{\mathrm{b}}$, and C.B. Becker ${ }^{\mathrm{b}}$ \\ aPennington Biomedical Research Center, 6400 Perkins Rd., Baton Rouge, LA 70808 \\ bTrinity University, One Trinity Place, San Antonio, TX 78212-2100
}

\title{
Abstract
}

Objective-The primary aim of this paper was to investigate moderators and predictors of response to two programs designed to reduce eating disorder risk factors in collegiate female athletes. This study served as an ancillary study to a parent trial that investigated the feasibility of an athlete modified cognitive dissonance-based program (AM-DBP) and an athlete modified healthy weight intervention program (AM-HWI).

Design-157 female collegiate athletes were randomized to either the AM-DBP or the AM-HWI program. Participants completed surveys at baseline, post-intervention, 6 weeks, and 1 year.

Methods-After classifying sports as either lean or non-lean, we investigated if sport type acted as a moderator of program response to AM-DBP and AM-HWI using ANOVAs. Next, we examined whether baseline thin-ideal internalization, weight concern, shape concern, bulimic pathology, dietary restraint, and negative affect acted as predictors of changes in bulimic pathology using linear regression models.

Results-Athletes in non-lean sports who received AM-DBP showed more improvement in negative affect versus non-lean sport athletes in AM-HWI. Higher baseline scores of bulimic pathology predicted greater response in bulimic pathology to both programs at 6-weeks. In contrast, athletes with higher dietary restraint and negative affect baseline scores showed decreased response to both interventions at 6-weeks. Finally, athletes with higher baseline shape concern showed a decreased response to the AM-HWI intervention at the post intervention time point.

Conclusion-Results from the present study indicate that lean/non-lean sport may not play a strong role in determining response to efficacious programs. Further, factors such as pre-existing bulimic pathology, dietary restraint, negative affect, and shape concern may affect general response to intervention versus specific responses to specific interventions.

\footnotetext{
(C) 2014 Elsevier Ltd. All rights reserved.

Corresponding Author: Tiffany M. Stewart, Ph.D., Pennington Biomedical Research Center, 6400 Perkins Rd., Baton Rouge, LA 70808, Phone: (225) 763-2554, Stewartm@pbrc.edu.

Publisher's Disclaimer: This is a PDF file of an unedited manuscript that has been accepted for publication. As a service to our customers we are providing this early version of the manuscript. The manuscript will undergo copyediting, typesetting, and review of the resulting proof before it is published in its final citable form. Please note that during the production process errors may be discovered which could affect the content, and all legal disclaimers that apply to the journal pertain.
} 


\section{Keywords}

disordered eating; female athletes; sport; prevention; intervention; moderators; predictors

Collegiate female athletes comprise a special population of college women who often are at elevated risk for engaging in disordered eating habits and developing eating disorders (EDs) when compared to the general population (Sundgot-Borgen \& Torstveit, 2004). Exposure to societal pressures to be thin, coupled with the demands of sport culture that focuses on body appearance, social comparison, body-revealing uniforms, and judging criteria, may result in a unique susceptibility to body image dissatisfaction and disordered eating (Greenleaf, Petrie, Carter, \& Reel, 2009). Many athletes also believe that reducing weight or body fat will enhance athletic performance, even in non-judged sports (Thompson \& Sherman, 2010).

As a result, many female athletes engage in restrictive eating patterns in order to reduce body weight and/or body fat (Hinton, 2005). However, female athletes often need to consume more calories than their non-athlete counterparts to sustain their active lifestyle. As such, it is not surprising that a recent study showed that 70 percent of female athletes in a college sample were not eating enough calories to meet their daily energy needs (Hinton, 2005). Female athletes who do not consume sufficient nutrient-rich calories to sustain their physical activity level are at a risk for developing the female athlete triad, which consists of inadequate energy availability, menstrual disorders, and diminished bone health (Manore, Kam, \& Loucks, 2007).

\section{Prevention of Eating Disorders}

Although more effective treatments for anorexia nervosa (AN) and bulimia nervosa (BN) have been developed, the success of treatment interventions for $\mathrm{AN}, \mathrm{BN}$, and eating disorder not otherwise specified (EDNOS; i.e., clinically significant eating disorders that do not meet criteria for $\mathrm{AN}, \mathrm{BN}$, or binge eating disorder (BED)) remains limited. For example, in a recent test of enhanced cognitive behavioral therapy, one of the most promising new eating disorder treatments for adults with AN, only $55 \%$ of participants in an open 2-country trial completed treatment and had minimal eating disorder psychopathology at the end of treatment (Fairburn et al., 2013); at 6 month follow-up in the same study the percentage with minimal eating disorder psychopathology dropped to $43 \%$. Research also has demonstrated that there are elevated mortality rates for AN, BN, and EDNOS (Crow et al., 2009). Given the lack of effective treatment interventions for a large portion of the ED population, increased attention has been placed on prevention programs.

Historically, prevention programs have consisted primarily of psychoeducation/didactic components (i.e., instructional, educational, passive participation). However, research indicates that interactive programs (i.e., require active participation) are more effective at reducing ED pathology and risk factors (Stice \& Shaw, 2004). Using this new paradigm, two dominant prevention strategies, both interactive programs, have emerged and received the most empirical support and independent replication: a cognitive dissonance-based 
prevention program (DBP) (Stice, Shaw, Becker, \& Rohde, 2008), and a healthy weight intervention program (HWI) (Stice, Chase, Stormer, \& Appel, 2001).

DBP incorporates the theory of cognitive dissonance, which states that discrepancies between an individual's beliefs and behavior lead to the alteration of beliefs in order to reduce psychological discomfort (Festinger, 1957). Thus, in the DBP program, participants engage in activities that reject the thin-ideal standard of female beauty promoted by western culture. This theoretically causes discomfort due to incongruence between any present thinideal internalization or body image concerns and the counter-attitudinal exercises. The psychological discomfort is then presumed to lead to a reduction in thin-ideal internalization. Based on Stice's dual pathway model, a decrease in thin-ideal internalization will lead to a decrease in both body dissatisfaction and negative affect, and ultimately, a decrease in ED pathology (Stice, 2001).

Alternatively, the HWI program endorses healthy changes in dietary intake and physical activity and aims to increase body satisfaction by teaching participants how to manage their weight in a healthy manner. HWI was developed by Stice and colleagues initially as a placebo control group against which to compare to DBP. When HWI produced positive effects, however, researchers began studying it as a potentially effective program in its own right. In HWI, participants evaluate eating and exercise habits via self-monitoring and then commit to small, manageable changes to diet and exercise during home assignments. Theoretically, once participants experience progress in changing unhealthy habits, they feel empowered to manage their weight for their body type. Both the behavioral change strategies and increased self-efficacy are presumed to contribute to decreased body dissatisfaction, negative affect, and ED pathology.

Both DBP and HWI programs have been shown to reduce eating disorder risk factors in randomized control trials conducted by independent researchers (Becker, Bull, Schaumberg, Cauble, \& Franco, 2008; Becker, Smith, \& Ciao, 2006; Becker et al., 2010; Green, Scott, Diyankova, Gasser, \& Pederson, 2005; Matusek, Wendt, \& Wiseman, 2004; McMillan, Stice, \& Rohde, 2011; Stice, Shaw, Burton, \& Wade, 2006; Stice, Rohde, Gau, \& Shaw, 2009; Stice, Marti, Spoor, Presnell, \& Shaw, 2008). Perhaps more importantly, they have been found to actually reduce the onset of EDs at 3-year follow-up. For example, Stice and colleagues (2008b) found that both programs reduced the onset of new eating disorder cases by approximately $60 \%$ relative to an assessment only control condition. Further, research also supports the viability of using peer-facilitators in a college-age sample of women (Becker et al., 2008; Becker et al., 2006; Becker et al., 2010). While DBP and HWI programs have yielded positive findings, more research is needed to determine whether or not these findings generalize to populations with specialized needs, such as female athletes.

Despite the fact that female athletes may be at a greater risk for disordered eating and development of the female athlete triad, few studies have examined eating disorder prevention programs with this population. One notable exception evaluated Athletes Targeting Healthy Exercise and Nutrition Alternatives (ATHENA), a sport-team centered, peer-led prevention program for female athletes (Elliot et al., 2004). In this program, participants completed activities that addressed the benefits of appropriate sport nutrition 
and exercise training over substance abuse and other unhealthy behaviors. Other facets of the program addressed self-esteem and depression. One of ATHENA's exercises asked participants to make public service announcements that discourage disordered eating habits (Elliot et al., 2004). A trial evaluating ATHENA against a usual-care control group demonstrated that athletes who received this program reported less use of diet pills and less new use of athletic/performance enhancing substances, as well as positive changes in dieting habits compared to those only receiving an educational pamphlet (control group) (Elliot et al., 2004).

More recently, Becker, McDaniel, Bull, Powell, and McIntyre, (2012) (parent trial for the present study) randomized female athletes to either a peer-led athlete modified dissonance based prevention program (AM-DBP) or an athlete modified healthy-weight intervention (AM-HWI). Participants showed statistically significant reductions in thin-internalization, dietary restraint, bulimic pathology, shape concern, weight concern, and negative affect at a 6-week follow-up in both programs. These results held for negative affect, bulimic pathology, and shape concern at a 1-year follow-up (Becker et al., 2012). Unexpectedly, this study also found that a clinically significant number of athletes receiving this intervention approached their head athletic trainer with concerns that they had developed part of the female athlete triad, which had not occurred prior to the intervention (Becker et al., 2012). With the development and evaluation of promising ED risk factor reduction programs, further research is needed to identify moderators and predictors of response to these programs in order to maximize potential benefits to participants.

\section{Identification of Moderators and Predictors of Program Response}

Identifying moderators and predictors of response to prevention strategies can provide useful information for improving intervention efficacy. A moderator may be considered an effect modifier or characteristic believed to be associated with variation in effect sizes. This variable influences the relationship between two variables in terms of magnitude of direction (Kazdin, 2003). Thus, moderators essentially aid in the identification of subgroups for which an intervention's, or in this case, a prevention program's, effects are stronger or weaker relative to the remainder of the sample (Stice, Marti, Shaw, \& O’Neil, 2008).

Like moderators, nonspecific treatment response predictors indicate who benefits from intervention/treatment, or under which conditions the intervention works best (Kraemer, Wilson, Fairburn, \& Agras, 2002). Both moderators and predictors are variables that can be measured before treatment and are associated with outcomes; however, predictors do not differ across treatment conditions while moderators change the magnitude or direction of the effects across treatments. Thus, moderators and predictors can help determine which participants should receive a particular intervention/treatment.

Identifying client characteristics that predict a better response to a certain treatment allows clinicians to match interventions to clients on a scientific basis, increasing the likelihood of successful outcome. We can similarly place participants in selected prevention strategies knowing certain characteristics may result in larger effects. Matching requires that we evaluate moderators of change in prevention programs, which will help identify the 
characteristics of both the population and intervention that yield the strongest or most successful outcome (Stice \& Shaw, 2004).

Several studies have evaluated moderators and predictors of eating disorder intervention/ prevention program response in adolescent and college age women. For instance, a metaanalysis of ED prevention programs (Stice \& Shaw, 2004) revealed that selected (i.e., targeting high risk individuals) versus universal (i.e., targeting both lower and higher risk members of a population) programs yielded larger effects. Additionally, Stice et al. (2008a) found that a DBP program had stronger effects on adolescent women with initially elevated body image distress, bulimic symptoms, and thin-ideal internalization, whereas, a HWI program had stronger effects on bulimic symptoms in adolescents with initially elevated body image distress, readiness to change, body mass, and emotional eating. Overall, both programs had stronger effects on high-risk rather than low-risk adolescents (Stice et al., 2008a). This may occur because high-risk participants are more likely to be motivated to engage in such a program, increasing potential benefits (Stice, Shaw, \& Marti, 2007). It should be noted, however, that in a more recent paper Muller \& Stice (2013) failed to find that baseline body dissatisfaction moderated intervention effects. Lastly, Stice, Rohde, Gau and Shaw (2012) found that negative affect reduced the effects of both the DBP and HWI. To date, research aimed at identifying moderators and predictors of response in prevention programs in special at- risk populations (e.g. female athletes) has been lacking. One reason for this is that, until recently, few studies have yielded positive effects on eating disorder risk factors in female athletes.

\section{Study Purpose and Hypotheses}

The primary aim of this study was to identify moderators and predictors of response to two eating disorder risk factor reduction programs in collegiate female athletes. Identification of such moderators and predictors could improve development of effective programming for the prevention of eating disorders in female athletes. Given this, the current study sought to investigate aspects of an athlete population as possible moderators and predictors of response to the AM-DBP and AM-HWI programs. First, we sought to investigate the type of sport as a possible moderator of response on all primary dependent variables for this study (i.e., bulimic pathology, thin-ideal internalization, shape concern, weight concern, dietary restraint, and negative affect). As such, sports in this study were divided into two categories consisting of lean versus non-lean sport (Thompson \& Sherman, 2010). These categories were created because some sports are thought to be under more pressure to be lean due to a variety of factors including but not limited to the nature of the sport, uniforms required, and degree of body scrutiny. It is of note that it is not believed that athletes in the "non-lean" category do not receive pressure to maintain a lean physique, but may experience this pressure to a lesser degree (e.g., gymnastics versus softball). Second, based on the general literature on eating disorder risk factors and past moderator studies, we examined baseline levels of the primary dependent variables (e.g., dietary restraint, shape concern, negative affect) in the parent study as potential predictors of response.

The parent study (Becker et al., 2012) found that both the AM-DBP and the AM-HWI programs improved dependent measures at post- intervention and follow-up time points (see 
results for additional details). In the present paper, we examined the hypothesis that sport type would moderate the prevention program effect on outcome variables. Based on observations from the parent study that some lean sport teams reported a preference for AMHW and/or found AM-DBP challenging, we hypothesized that lean sports would show greater response to AM-HWI than AM-DBP whereas non-lean sports (which appeared more open to AM-DBP) would show greater responsiveness to AM-DBP. With regards to predictors we tested the hypotheses that baseline thin-ideal internalization, shape concern, weight concern, dietary restraint, and negative affect would predict change scores in bulimic pathology at post-intervention and a 6-week follow-up. Regarding more specific hypotheses, we found limited literature available to guide predictions. Based on Stice et al. (2012), Stice et al. (2008a) and Muller and Stice (2012) we hypothesized that elevated bulimic pathology would increase response to both programs and elevated levels of negative affect would decrease response to both programs. We also hypothesized that higher levels of thin-ideal internalization would predict greater responsiveness to AM-DBP. Because past research has found contradictory findings with regards to body dissatisfaction and there is no literature to our knowledge to guide specific hypotheses with respect to dietary restraint, these analyses were exploratory.

\section{Methods}

\section{Participants}

One hundred and seventy seven athletes participated in the actual programs; the sample for this study consisted of 157 (see Becker et al., 2012 for additional information including CONSORT flow diagram), primarily Caucasian (75.8\%), university female athletes participating in 9 different varsity sports from a highly competitive NCAA Division III program in the United States. Sports included basketball $(n=18)$; swimming and diving ( $n=$ $26)$; softball $(n=15)$; tennis ( $n=14)$; cross country, $(n=18)$; soccer $(n=23)$; volleyball $(n$ $=14)$; golf $(n=4)$; track and field $(n=12)$; and 13 members of a varsity cheerleading squad. Participants ranged in age from 18 to 22 years $(M=18.94, S D=1.04)$. Body mass index (BMI) ranged from 16.0 to $37.78 \mathrm{~kg} / \mathrm{m}^{2}(M=22.2, S D=2.4)$. Those meeting probable criteria for a clinically significant eating disorder, as assessed by the Eating Disorder Examination Questionnaire (EDE-Q) (Fairburn \& Beglin, 1994) were removed from the sample as both programs focused on prevention and not treatment (see Becker et al., 2012 for additional information).

\section{Study Design}

Becker et al. (2012) conducted a pilot study (parent study) to determine the feasibility and promise of two programs, an athlete modified cognitive dissonance-based prevention program (AM-DBP) and an athlete modified healthy weight prevention program (AMHWI), aimed at reducing ED risk factors in female collegiate athletes. During this study, the department of athletics requested that all athletes receive an active intervention in the study; thus, the parent study did not include a no-treatment control group. Each team was split into two groups with half of each sport team being randomized to either the AM-DBP program or the AM-HWI program. Details on the methods of the parent study may be found elsewhere (Becker et al., 2012). 
The present study serves as an ancillary study to the parent study, and is the first investigation of possible predictors and moderators of response to prevention programming in female athletes. This type of investigation was not previously possible in the literature on female athletes because previous programs were largely unsuccessful in changing eating disorder related dependent variables. The present study utilized the baseline and postintervention data to test for a moderator effect for sport team type (lean versus non-lean), because the strongest effects were found post-intervention in the parent study. The present study also tested for predictor effects for baseline thin-ideal internalization, weight concern, shape concern, and negative affect on change scores for bulimic pathology at both postintervention and 6-week follow-up.

\section{Procedure}

All athletes participated in the intervention(s) but participation in the study was voluntary, which consisted of completing assessment instruments only. Therefore, there were athletes who received the intervention but did not participate in the study. This procedure was approved by the Trinity University institutional review board (IRB) and has been utilized in previous trials with other populations (Becker et al., 2008). Coaches were not allowed to ask athletes to participate in the study nor were they present during any of the program sessions or assessments in order to prevent problems with coercion. Finally, in accordance with the IRB, athletes signed consent forms in order to participate in the study. Participants completed measures assessing thin-ideal internalization, bulimic pathology, dietary restraint, body dissatisfaction, and negative affect at 4 different times (baseline, post-intervention, 6week follow-up, 1-year follow-up). Additional detail regarding the procedure of the parent study may be found elsewhere (Becker et al., 2012).

\section{Interventions}

Interventions consisted of three sessions each lasting approximately $60-80$ minutes over a three-week period. Consistent with a participatory model, all groups were peer-led by female athletes.

AM-DBP-The AM-DBP manual was adapted from the DBP manual developed by Stice and Presnell (2007). As noted above DBP is highly interactive versus didactic and includes extensive group discussions, writing activities, homework, and role-plays. For example, group leaders do not describe the thin-ideal to participants and provide a list of the costs of pursuing it; rather participants as a group define the thin-ideal and then individually (writing exercise) create a list of costs which are then shared with the larger group to create a group list. In comparison to traditional DBP, AM-DBP included information on the female athlete triad and targeted body image pressures that athletes faced in their specific sport. Specifically, this program focused on the sport-specific thin-ideal of female beauty (i.e., what a certain sport views as the ideal body type, and/or the body type defined by athletes for their respective sports) in addition to the thin-ideal standard of female beauty. This program consisted of three sessions which focused on examining the costs of pursuing thin ideals, mirror exercises, information on the female athlete triad/ways to avoid it, role play designed to speak out against the thin ideal, and homework assignments. Further details may be found in the parent study paper (Becker et al., 2012). 
AM-HWI-The AM-HWI manual was based on the HWI manual developed by Stice and Presnell (2007). As with AM-DBP, the manual is designed to facilitate group discussions and participation by group members. The AM-HWI also included information on the female athlete triad as well as discussion of the sport-specific thin-ideal and the athlete-specific healthy-ideal. This manual also included three sessions and recognized that athletes do not always have to increase their level of activity and may actually benefit from a reduction in training - particularly for athletes who are exercising more than recommended by their coaches. This manual included additional focus on increasing nutritional density (i.e., eating foods that provide a high degree of nutrients in proportion to calories), reducing the consumption of highly processed food, and managing sleep in order to communicate that the goal was health and not the pursuit of either thin-ideal. The manual also asked participants to explore ways to improve performance other than weight-loss. Finally, this manual asked athletes to think about changes in their behavior that occurred during and off-season. Further details may be found in the parent study paper (Becker et al., 2012).

\section{Assessment Measures}

Thin-ideal internalization-Thin-ideal internalization was measured using the Ideal Body Stereotype Scale-Revised (IBSSR) (Stice \& Agras, 1998). This measure is a 10-item questionnaire that asks participants to rate to what extent they agree or disagree (strongly disagree $=1$, strongly agree $=5$, scale range: $1-5)$ with certain statements promoting the thin-ideal standard of female beauty (e.g., slim women are more attractive). This scale has demonstrated adequate internal consistency $(\alpha=.89)$ and test-retest reliability $(r=.63)$ (Stice $\&$ Agras, 1998). Internal consistency in the present sample was consistent with previous research $(\alpha=.86-.92)$.

Bulimic pathology-Bulimic pathology was measured by taking a composite score from the diagnostic items from the Eating Disorders Examination-Questionnaire (EDE-Q) (Fairburn \& Beglin, 1994). The 10 diagnostic items assess the degree to which participants engaged in eating disorder behaviors over the past 28 days. This measure has been shown to be reliable and valid (Fairburn \& Beglin, 1994). Internal consistency in the present sample was consistent with previous research $(\alpha=.83-.85)$.

Dietary restraint-Dietary restraint was assessed using the Dutch Restrained Eating Scale (DRES) (Van Strien, Fritjers, Bergers, \& Defares, 1986). This 10-item questionnaire asks participants to report how often $(1=n e v e r, 5=$ always, scale range $=1-5)$ they engage in restrained eating behaviors (e.g., Do you deliberately eat less in order not to become heavier?). Although research has shown that measures of dietary restraint are not good at measuring actual dietary intake (Stice, Fisher, \& Lowe, 2004), they have found that these measures are effective at predicting bulimic pathology (Stice et al., 2004), which is why we chose to utilize this measure in the present study. Research supports the internal consistency $(a=.95)$ and predictive validity of the DRES (Stice \& Agras, 1998; Van Strien et al., 1986). Internal consistency in the present sample was consistent with previous research $(\alpha=.93-$. 94). 
Body dissatisfaction-Body dissatisfaction was evaluated using the Shape and Weight Concern subscales of the EDE-Q (Fairburn \& Beglin, 1994). The 8-item Shape Concern subscale assesses how frequently participants are concerned about their shape over the past 28 days (e.g., has your shape influenced how you think about (judge) yourself as a person?). The 5-item Weight Concern subscale assessed how often participants are concerned about their weight over the past 28 days (e.g., how dissatisfied have you been with your weight?). Both subscales have demonstrated good internal consistency at baseline (shape concern, a $=.93$; weight concern, $\alpha=.89$ ) and 2-week test-retest reliability (shape concern, $r=.94$; weight concern, $r=.92$ ) (Luce, Crowther, \& 1999). Internal consistency in the present sample was consistent with previous research (shape concern, $a=.92-.94$; weight concern, $a=.85-.89)$.

Negative affect-Negative affect was measured using the Fear, Guilt, and Sadness subscales from the Positive Affect and Negative Affect Schedule-Revised (PANAS-X; Watson \& Clark, 1992). These 17 items asses to what degree ( $1=$ not at all, $5=$ extremely; scale range: 1-5) participants are feeling certain emotions (e.g., nervous, guilty, afraid) over the past few weeks. This scale has demonstrated good internal consistency $(a=.95)$, convergent validity, and predictive validity for bulimic symptom onset (Stice \& Agras, 1998; Watson \& Clark, 1992). Internal consistency in the present sample was consistent with previous research $(\alpha=.91-.93)$.

\section{Analytic Plan}

For the purposes of analyses, teams were classified into two groups: lean and non-lean sport. We chose not to use more specific categories (e.g. endurance, aesthetic, ball game) because we lacked sufficient statistical power to break the sample into these smaller categories. Thus, using criteria largely derived from Thompson and Sherman (2010), we classified basketball, softball, soccer, volleyball (although the evolution of volleyball uniforms to more revealing clothing may now make it difficult to classify), and golf as non-lean sports. Swimming, diving, tennis, cross -country, track, and cheerleading were all classified into the lean sport category. The most difficult team to classify was tennis. As noted by Thompson and Sherman (2010), no matter what classification system is used, some sports do not easily fit into categories. On the one hand, tennis can be viewed as either a technical or ball game sport or even an endurance sport given the length of many matches. On the other hand, in recent years, female tennis players have been under increasing pressure to maintain a certain physique that appears to go beyond that of other ball game sports such as soccer (see Harris, 2000; Seles, 2009). An example of this can be seen in the case of Taylor Townsend, who lost funding to play in the US Open and was pressured by the USTA to not attend on the basis of her weight, despite being the number one ranked junior tennis player in the world (http://tennis.si.com/2012/09/07/taylor-townsend-usta-controversy/).

Separate Analyses of Covariance (ANCOVAs) models were conducted to investigate sport type as a potential moderator of outcome variables (thin-ideal internalization, shape concern, weight concern, negative affect, bulimic pathology) at post-intervention and 6-week followup. Missing data points were handled with PROC mixed procedure with maximum likelihood imputation procedures in SAS. For each outcome, sport type, intervention, and 
the interaction between sport type and intervention were entered in the model, controlling for the baseline measure of the outcome variable. Effect size was estimated using a $d$ change where $d$ is Cohen's $d$, which is mean difference divided by pooled standard deviation. We measured $d$ for each of the two levels of moderator (lean, non-lean sport type) and compared them.

To test whether baseline variables were predictors at post-intervention and 6-week followup, linear regression models were constructed for bulimic pathology outcome. The dependent variable consisted of the change score at the post-intervention time point (postintervention - baseline) and the independent variable consisted of the baseline value of the bulimic pathology, the potential predictor (baseline scores of other response variables), and the sport type (lean, non-lean). The final model was selected using a backward selection technique to remove non-significant variables at the .30 level. The same regression analyses were performed by each intervention arm and at 6-week follow-up as well. Cohen's $f^{2}$ was used to estimate effect size for the multiple regressions. It is calculated as the ratio between $\mathrm{R}^{2}$ and $\left(1-\mathrm{R}^{2}\right)$. Because the eating disorder pathology composite was skewed, we normalized using a square root transformation. Skewed PANAS data was normalized using a logarithmic transformation. All statistical analyses were performed using SAS 9.2.

\section{Results}

\section{Summary of Results from Parent Study}

All analyses were conducted using intent-to-treat using hierarchical linear modeling with post-hoc t-tests. Results indicated that compared to baseline, both AM-DBP and AM-HWI reduced thin-ideal internalization $(\mathrm{p}<.001)$, dietary restraint $(\mathrm{p}<.05)$, bulimic pathology $(\mathrm{p}$ $<.01)$, shape concern $(\mathrm{p}<.05)$, and negative affect $(\mathrm{p}<.01)$ at post-intervention. Both groups showed a reduction in thin-ideal internalization $(\mathrm{p}<.05)$, dietary restraint $(\mathrm{p}<.001)$, bulimic pathology $(\mathrm{p}<.001)$, shape concern $(\mathrm{p}<.001)$, weight concern $(\mathrm{p}<.01)$, and negative affect $(\mathrm{p}<.01)$ at 6 -week follow up. Reductions in bulimic pathology $(\mathrm{p}<.05)$, shape concern $(\mathrm{p}<.05)$, and negative affect $(\mathrm{p}<.05)$ were retained at 1 -year compared to baseline. (See Becker et al., 2012 for additional detail).

\section{Moderator Effect of Sport team Type}

In the test of moderation effects on outcomes, only one significant finding emerged. Sport type interacted with intervention in predicting changes in negative affect at postintervention, $F(1,144)=5.64, p=.019$. Among participants on lean teams, there was no difference on change in negative affect at post-intervention between the two intervention groups (AM-DBP lsmean $=-0.18$; AM-HWI lsmean $=-0.19 ; p=.80 ; d=0.05)$. In contrast, participants on non-lean teams had a significant reduction in negative affect at postintervention in AM-DBP versus those in AM-HWI (AM-DBP lsmean $=-0.32$; AM-HWI lsmean $=-0.07 ; p<.01 ; d=0.64)$.

\section{Predictors of Treatment Outcome}

Table 1 shows the variables that were statistically significant predictors of changes in bulimic pathology at post-intervention and 6-week follow-up. The effect size Cohen's $f^{2}$ was 
0.20 and 0.19 overall at post and 6-week follow-up, which represent moderate to large effect sizes. Cohen's $f^{2}$ was in similar range for both the AM-DBP and AM-HWI interventions ( 0.25 and 0.33 for AM-DBP, 0.25 and 0.16 for AM-HWI at post and 6-week follow-up).

With respect to specific findings that held at both post-intervention and 6-week follow-up, participants who reported more bulimic pathology at baseline had greater reductions in bulimic pathology at post-intervention and 6-week follow-up, for the overall sample (i.e., all participants) and in both the AM-DBP and AM-HWI intervention groups $(p<.01)$. Baseline values of dietary restraint also predicted changes in bulimic pathology scores at postintervention and 6-week follow-up for all participants and in the AM-DBP intervention group, after controlling for baseline bulimic pathology (all $p$ 's $<.05$ ). In contrast to the finding with bulimic pathology, however, all participants with high baseline dietary restraint scores evidenced decreased reduction in bulimic pathology.

Baseline shape concern predicted changes in bulimic pathology only at post intervention for the total sample and for participants in AM-HWI, after controlling for bulimic pathology ( $p$ 's $<.05$ ). Thus, as a whole, participants with initial elevated body shape concern showed a decreased response and this finding held specifically for the AM-HWI intervention.

Lastly, baseline negative affect was positively related with change in bulimic pathology at 6week follow-up for all participants $(p<.05)$, indicating that increased negative affect was associated with reduced improvement. It should be noted, however, that this finding did not hold for either of the specific interventions.

\section{Discussion}

Previous research (Becker et al., 2012) provided preliminary support for two programs (i.e., AM-DBP and AM-HWI) in reducing ED symptoms and risk factors in female athletes.

Results indicated that both programs reduced all assessed ED risk factors, (i.e., thin-ideal internalization, bulimic pathology, negative affect, weight concern, and shape concern) at 6 weeks; at 1-year follow-up, effects remained for shape concern, bulimic pathology, and negative affect (Becker et al., 2012). To supplement this previous research, the present study investigated potential moderators and predictors of response to these two programs.

We hypothesized that participants in lean sports would respond better to AM-HWI and participants in non-lean sports would respond better to AM-DBP based on observations of preferences in the parent trial. Results of the moderator analysis in the present study largely failed to support our hypotheses and generally indicated that sport type was not a moderator of response. More specifically, for all but one dependent variable, there were no differences for lean and non-lean sports. This suggests that for the overwhelming majority of assessed risk factors both programs worked equally well for both groups. Importantly, given that this is the first evaluation of moderator effects, results need to be replicated, ideally with a larger sample and with more precise categories of sports given that lean versus non-lean categorization may not be fine-tuned enough to detect more specific differences between sport types. The present study was unable to investigate more specific categories due to limitations in statistical power and represents a first step regarding investigation of 
moderators. Thus, further research should investigate other ways of classifying sports beyond the lean/non-lean categories. It also would be helpful to investigate the moderating effects of sport status (i.e., lean or non-lean) when the study design includes a no-treatment control group as that study design might yield different results.

Despite these limitations, the current results do support the standard method of delivering these types of programs to female athletes. More specifically, both the parent study and the ATHENA program (Elliot et al., 2004) delivered the same intervention to participants across a wide array of sports. Thus, although we recommend that the findings of the present study be replicated and that researchers test sport type as potential moderator in future research, for the time being the limited existing data support providing the same programming to different sport types. We should note that one reason for the lack of difference may be a general cultural trend to increasingly expect all athletes to achieve a particular athletic looking physique even in sports where no such pressure previously existed.

Results of the predictor analyses indicated that the effects of both programs on bulimic pathology were significantly stronger for athletes who reported greater bulimic pathology at baseline. This effect is consistent with our hypothesis and in line with prior non-athlete trials demonstrating that ED prevention programs have greater impact on those who have elevated risk factors (Muller \& Stice, 2013; Stice et al., 2008a). It has been hypothesized that individuals with elevated symptoms may be more likely to engage in a program because they may be more motivated to gain relief from symptoms (Muller \& Stice, 2013; Sticeet al., 2008a). It is unclear, however, if this interpretation applies to the present study given that participants were required by athletics to participate in the program (but not the study which consisted only of completing the questionnaires). Thus, in contrast to research by Stice and colleagues (2008a), participants did not self-select into the programs. An alternative explanation is that those with elevated scores have greater range for improvement as well as reduced potential for floor effects. That explanation remains a possibility in the present trial, although it is inconsistent with what we found with other variables. We should note that the attrition did not seem to drive results as $96 \%$ of the AM-DBP sample was retained at 6-week follow-up; similarly in AM-HWI the retention rate was 93\%.

The bulimic pathology results might suggest to some that prevention efforts should be focused exclusively on those already reporting elevated levels of disordered eating. Yet, because findings may result more from measurement issues (e.g., floor effects) in lower-risk participants and because such an approach runs counter to strong feedback we have received from athletics departments around the country, we caution against this conclusion. More specifically, many athletics departments report wanting to use programs like the ones in the present study to meet multiple needs (e.g., team building, leadership opportunities for athletes), not just reduce eating disorder risk factors. In addition, as noted by Kazdin and Blase (2011) although the primary goal of traditional intervention research has been to strategies that yield the largest effect sizes, this may not be the best way to yield the largest effects across a population. For instance, a program with a very reliable yet weaker effect size that can be broadly disseminated because it meets multiple needs and gains greater buyin from decision makers may actually yield a greater impact on a population than one with a large effect size that is to only be delivered to a few people. Further, given that a mediation 
analysis of the ATHENA program indicated that social norms mediated effects of the program, prevention programs may lose efficacy if they are implemented only with selected athletes (Ranby et al., 2009)

Also partially supportive of our hypotheses, athletes with elevated baseline negative affect reported less improvement in their bulimic pathology symptoms at 6-week follow-up but not post-intervention. This finding held for the overall sample, but not the specific interventions. Regardless, it contributes to a growing literature suggesting that negative affect may affect the outcome of both prevention (Stice et al., 2012) and treatment of eating disorders (e.g., LeGrange, Crosby, \& Lock, 2008). This finding additionally is consistent with research showing that substance prevention programs are less effective for those with higher levels of depression (Amaro, Blake, Schwartz, \& Flinchbaugh, 2001). It is unclear why this result did not emerge until follow-up. One possible reason may relate to the fact that the sample continued to improve over the 6-week follow-up period. For instance, the effect size for AM-DBP from baseline to 6-week follow-up increased to .40 compared to a .28 at postintervention. Thus, one interpretation is that negative affect did not significantly affect athletes' ability to engage with the material during the active intervention but did affect their ability to continue to use and further benefit from the tools they learned after the interventions ended. Given that negative affect is associated with significant cognitive changes in memory, it also might be that over time athletes who are high in negative affect are less able to recall the positive experience of the group and better recall negative body image events in their lives thus diluting the effects of the intervention. Given that this effect only emerged at one time point and for the entire sample but not for the individual interventions, a first critical step will be to determine if this finding replicates in future trials.

We also found that athletes with elevated baseline dietary restraint scores largely showed attenuated response at both post-intervention and follow-up. This was one of the more robust findings, but also one of the most difficult to interpret given that the general literature on dietary restraint is problematic. Dietary restraint is a well-recognized, very robust and consistent risk factor for the development of eating disorders (Stice, 2001; Stice et al., 2004), which is why dietary restraint measures continue to be included in prevention trials, including the parent trial. Yet, it is also clear that dietary restraint does not in fact measure actual restriction in eating behavior or decreased caloric intake (Stice et al., 2004), making it unclear what in fact such measures actually assess. It may be that elevated dietary restraint is an indicator of a general desire to eat less, versus actual success with eating less. Thus, one possible interpretation is that athletes who had a strong desire to eat less responded less well to the interventions because both included interactive components aimed at teaching female athletes about the need for some of them to eat more to avoid the female athlete triad. Thus, the interventions may not be sufficiently potent for participants who are highly invested in decreasing dietary intake. For instance, it might be that those who are highly invested in restriction need a larger dose of the intervention in order to benefit or would benefit from a program run by more experienced clinicians. Given that participants with increased bulimic symptoms responded better to the interventions, it also might be that those who are invested in trying to restrict but have not succeeded, and therefore are not experiencing consequences (i.e., symptoms), have a harder time accepting the messages of the programs. We should note that determining why high dietary restraint participants did not respond as well to the 
interventions is challenging given that, as noted above, it remains unclear what this measure actually assesses and given that this is the very first study to investigate predictors. Thus, one major takeaway from the present study is a need for future research to investigate a) whether the dietary restraint predictor findings of the present study holds $b$ ) what construct is really being operationalized with the dietary restraint measures in female athletes so that such findings can be better understood, and c) changes that can be implemented in the interventions to better address the need of those who endorse high levels of dietary restriction.

Consistent with a mixed literature on the moderator effects of body dissatisfaction, we found mixed effects on our two measures of body dissatisfaction (i.e., weight concern and shape concern). Weight concern was the only variable that did not significantly predict response to either program, whereas shape concern predicted response overall and to AM-HWI, but only at post-intervention. We speculate that this finding may be reflective of body image in athletes being less focused on a traditional thin ideal, and more focused on a complex, sportspecific thin ideal. Although this varies somewhat depending on sport type, for athletes, shape is often a much more salient construct than simply low weight. For example, talk in the locker room (reported by athletes during the course of intervention in the parent study) tended to focus more on shape (including body fat) issues than weight per se. One hypothesis as to why this might be is the visual nature of locker rooms and the ability to compare body appearance with their peers.

This study had a number of limitations. First, it was constrained by the limitations of the original parent study. For instance, the study has increased risk of spillover effects because individuals versus teams were randomized to the interventions. We are currently conducting a follow-up study to address this design limitation. The study is also limited by exclusive reliance on self-report measures, and by the lack of a no-treatment control group. In addition, the study also has potential limited generalizability in that it was conducted on a Division III university campus. Although the teams are highly competitive within this division, athletes who are in a more competitive situation might respond differently as performance level has been shown to increase eating disorder risk. Thus, findings may be very different when more elite athletes are studied. In the above mentioned follow-up study, we are working with a broader range of performance-levels. Readers who are interested in a more detailed discussion of limitations of the parent study should refer to Becker et al. (2012).

This study also had additional limitations that were unique to the present study. For instance, secondary to lack of statistical power, we were unable to investigate whether or not more specific sport types (e.g., aesthetic versus endurance versus technical) had a moderating effect, and instead had to use the broader categories of lean versus non-lean sports. Although our non-lean category was relatively homogeneous (i.e., consisting mostly of ball game sports and one technical sport), the lean category was much more variable which may have influenced the results, or lack thereof, in the present analyses. Further, tennis was not easily classified in either category in our opinion, and our decision to place it in the lean category added variability to this category. Thus, this should be viewed as a limitation. Future

Psychol Sport Exerc. Author manuscript; available in PMC 2015 November 01. 
research with larger samples should investigate more precise sport categories and even individual sports' moderating effects.

In summary, to our knowledge, this is the first study to investigate moderators and predictors of eating disorder risk factor reduction program response in female collegiate athletes. Taken overall, results from the present study indicate that lean/non-lean sport may not play as strong a role in determining response to efficacious programs as expected. As noted above, however, more research is needed before this can be stated confidently. Similarly, we did not find large differences between the interventions with respect to predictors. This suggests that factors that are either associated with improved or decreased response to one intervention also affected response to the other intervention. If these findings hold in future research, then this might indicate that some factors (e.g., pre-existing bulimic pathology, dietary restraint, negative affect, and shape concern) may affect general response to intervention versus specific responses to specific interventions. Although the results of the present study were not entirely conclusive, they provide the first steps and rationale for future investigations on this line of research. In terms of future directions, researchers should examine the efficacy of these programs using randomized control trial designs with waitlist/assessment only controls so that it can be determined if these interventions will actually prevent eating disorders in athletic populations. In addition, the findings from the present study need to be replicated and extended, ideally via the research design mentioned above, using large samples. Large samples will be critical in providing sufficient statistical power to examine important additional potential moderators of program effects such as actual sport type and to ideally determine factors that moderate true prevention effects (versus reductions in risk factors). If findings from the present study are replicated, this may suggest that programs that work will tend to work for a wide array of sport types.

\section{Acknowledgments}

This study was supported by the National Institutes of Health: NIMH 077659.

\section{References}

Amaro H, Blake SM, Schwartz PM, Flinchbaugh LJ. Developing theory-based substance abuse prevention programs for young adolescent girls. Journal of Early Adolescence. 2001; 21(3):256293.10.1177/0272431601021003002

Becker CB, Bull S, Schaumberg K, Cauble A, Franco A. Effectiveness of peer-led eating disorders prevention: A replication trial. Journal of Consulting and Clinical Psychology. 2008; 76(2):347354. [PubMed: 18377130]

Becker CB, McDaniel L, Bull S, Powell M, McIntyre K. Can we reduce eating disorder risk factors in female college athletes? A randomized exploratory investigation of two peer-led interventions. Body Image. 2012; 9(1):31-42. [PubMed: 22019502]

Becker CB, Smith LM, Ciao AC. Peer-facilitated eating disorder prevention: A ramdomized effectiveness trial of cognitive dissonance and media advocacy. Journal of Counseling Psychology. 2006; 53(4):550-565.

Becker CB, Wilson C, Williams A, Kelly M, McDaniel L, Elmquist J. Peer-facilitated cognitive dissonance versus healthy weight eating disorders prevention: A randomized comparison. Body Image. 2010; 7(4):280-288. [PubMed: 20638351] 
Crow SJ, Peterson CB, Swanson SA, Raymond NC, Specker S, Eckert ED, Mitchell JE. Increased mortality in bulimia nervosa and other eating disorders. The American Journal of Psychiatry. 2009; 166(12):1342-1346. [PubMed: 19833789]

Elliot DL, Goldberg L, Moe EL, Defrancesco CA, Durham MB, Hix-Small H. Preventing substance use and disordered eating: initial outcomes of the ATHENA (athletes targeting healthy exercise and nutrition alternatives) program. Archives of Pediatrics and Adolescent Medicine. 2004; 158(11): 1034-1039.

Fairburn CG, Beglin SJ. Assessment of eating disorders: interview or self-report questionnaire? The International Journal of Eating Disorders. 1994; 16(4):363-370. [PubMed: 7866415]

Fairburn CG, Cooper Z, Doll HA, O’Connor ME, Palmer RL, Dalle Grave R. Enhanced cognitive behavior therapy for adults with anorexia nervosa: A UK-Italy study. Behaviour Research and Therapy. 2013; 51:R2-R8.10.1016/j.brat.2012.09.010 [PubMed: 23084515]

Festinger, L. A theory of cognitive dissonance. Stanford University Press; 1957. p. 1-280.

Green M, Scott N, Diyankova I, Gasser C, Pederson E. Eating disorder prevention: An experimental comparison of high level dissonance, low level dissonance, and no-treatment control. Eating Disorders: Journal of Treatment and Prevention. 2005; 13:157-169.

Greenleaf C, Petrie TA, Carter J, Reel JJ. Female collegiate athletes: Prevalence of eating disorders and disordered eating behaviors. Journal of American College Health. 2009; 57:489-496. [PubMed: 19254889]

Harris MB. Weight concernt, body image, and abnormal eating in college women tennis players and their coaches. International Journal of Sport Nutrition. 2000; 10(1):1-15.

Hinton, P. Running on empty. Training and conditioning. 2005. Retrieved from http://www.trainingconditioning.com/2007/03/running_on_empty.html website

Kazdin, A., editor. Research Design in Clinical Psychology. 4. Boston, MA: Allyn \& Bacon; 2003.

Kazdin AE, Blasé SL. Rebooting psychotherapy research and practice to reduce the burden of mental illness. Perspectives on Psychological Science. 2011; 6:21-37.

Kraemer HC, Wilson GT, Fairburn CG, Agras WS. Meditators and moderators of treatment effects in randomized clinical trials. Arhives of General Psychiatry. 2002; 59(10):877-883.

Le Grange D, Crosby RD, Lock J. Predictors and moderators of outcome in family-based treatment for adolescent bulimia nervosa. Journal of the American Academy of Child \& Adolescent Psychiatry. 2007; 47(4):464-470. [PubMed: 18388765]

LeMoult, J.; Castonguay, LG.; Joorman, J.; McAleavey, A. Depression. In: Castonguay, LG.; Oltmanns, TF., editors. Psychopathology: From Science to Clinical Practice. New York: Guildford Press; 2012. p. 17-61.

Luce KH, Crowther JH. The reliability of the Eating Disorder Examination-Self-report Questionnaire version (EDE-Q). International Journal of Eating Disorders. 1999; 25:349-351. [PubMed: 10192002]

Manore MM, Kam LC, Loucks AB. The female athlete triad: components, nutrition issues, and health consequences. Journal of Sports Sciences. 2007; 25:S61-S71. [PubMed: 18049984]

Matusek JA, Wendt SJ, Wiseman CV. Dissonance-based thin-ideal and didactic healthy behavior eating disorder prevention programs: Results from a controlled trial. International Journal of Eating Disorders. 2004; 36:376-388. [PubMed: 15558649]

McMillan W, Stice E, Rohde P. High-and low-level dissonance-based eating disorder prevention programs with young women with body image concerns: An experimental trial. Journal of Consulting and Clinical Psychology. 2011; 79:129-134. [PubMed: 21261438]

Muller S, Stice E. Moderators of the intervention effects for a dissonance-based eating disorder prevention program; results from an amalgam of three randomized trials. Behaviour Research and Therapy. 2013; 51:128-133. [PubMed: 23337181]

Ranby KW, Aiken LS, Mackinnon DP, Elliot DL, Moe EL, McGinnis W, Goldberg L. A mediation analysis of the ATHENA intervention for female athletes: Prevention of athletic enhancing substance use and unhealthy weight loss behaviors. Journal of pediatric psychology. 2009; 34(10): 1069-1083. [PubMed: 19386771]

Seles, M. Getting a grip: On my body, my mind, my self. New York: Penguin Group; 2009. 
Steinhausen HC. The outcome of anorexia nervosa in the 20th century. The American Journal of Psychiatry. 2002; 159(8):1284-1293. [PubMed: 12153817]

Stice E. A prospective test of the dual-pathway model of bulimic pathology: mediating effects of dieting and negative affect. Journal of Abnormal Psychology. 2001; 110(1):124-135. [PubMed: 11261386]

Stice E, Shaw H, Burton E, Wade E. Dissonance and healthy weight eating disorder prevention programs: A randomized efficacy trial. Journal of Consulting and Clinical Psychology. 2006; 74(2):263-275. [PubMed: 16649871]

Stice E, Rohde P, Gau J, Shaw H. An effectiveness trial of a dissonance-based eating disorder prevention program for high-risk adolescent girls. Journal of Consulting and Clinical Psychology. 2009; 77:825-834. [PubMed: 19803563]

Stice E, Agras WS. Predicting onset and cessation bulimic behaviors during adolescence: A longitudinal grouping analysis. Behavior Therapy. 1998; 29:257-276.

Stice E, Chase A, Stormer S, Appel A. A randomized trial of a dissonance-based eating disorder prevention program. International Journal of Eating Disorders. 2001; 29(3):247-262. [PubMed: 11262503]

Stice E, Marti N, Shaw H, O’Neil K. General and program-specific moderators of two eating disorder prevention programs. International Journal of Eating Disorders. 2008; 41(7):611-617. [PubMed: 18528875]

Stice E, Marti CN, Spoor S, Presnell K, Shaw H. Dissonance and healthy weight eating disorder prevention programs: long-term effects from a randomized efficacy trial. Journal of Consulting and Clinical Psychology. 2008; 76(2):329-340. [PubMed: 18377128]

Stice, E.; Presnell, K., editors. The Body Project. New York: Oxford University Press; 2007.

Stice E, Rohde P, Gau J, Shaw H. Effect of a dissonance-based prevention program on risk for eating disorder onset in the context of eating disorder risk factors. Prevention Science. 2012; 13(2):129139.10.1007/s11121-011-0251-4 [PubMed: 21975593]

Stice E, Shaw H. Eating disorder prevention programs: a meta-analytic review. Psychological Bulletin. 2004; 130(2):206-227. [PubMed: 14979770]

Stice E, Shaw H, Becker CB, Rohde P. Dissonance-based Interventions for the prevention of eating disorders: using persuasion principles to promote health. Prevention Science. 2008; 9(2):114-128. [PubMed: 18506621]

Stice E, Shaw H, Marti CN. A meta-analytic review of eating disorder prevention programs: encouraging findings. Annual Review of Clinical Psychology. 2007; 3:207-231.

Sundgot-Borgen J, Torstveit KT. Prevalence of eating disorders in elite female athletes. Clinical Journal of Sport Medicine. 2004; 14:25-32. [PubMed: 14712163]

Thompson, RA.; Sherman, RT., editors. Eating Disorders in Sport. New York: Taylor and Francis Group; 2010.

Van Strien T, Fritjers JER, Bergers GPA, Defares PB. The Dutch Eating Behavior Questionnaire (DEBQ) for assessment of restrained, emotional, and external eating behavior. International Journal of Eating Disorders. 1986; 5:295-315.

Watson D, Clark DM. Affects separable and inseparable: On the hierarchical arrangement of the negative affect. Journal of Personality and Social Psychology. 1992; 62:489-505. 


\section{Highlights}

1. Both athlete modified dissonance (DBP) and healthy weight (HWI) programs reduce ED symptoms and risk factors

2. Moderators and predictors of response to these programs were investigated

3. Sport type (lean/non-lean) was not a moderator of response

4. Effects of both programs on bulimic pathology were stronger for athletes with higher baseline bulimic pathology

5. Negative affect and elevated dietary restraint may attenuate outcome of ED prevention 


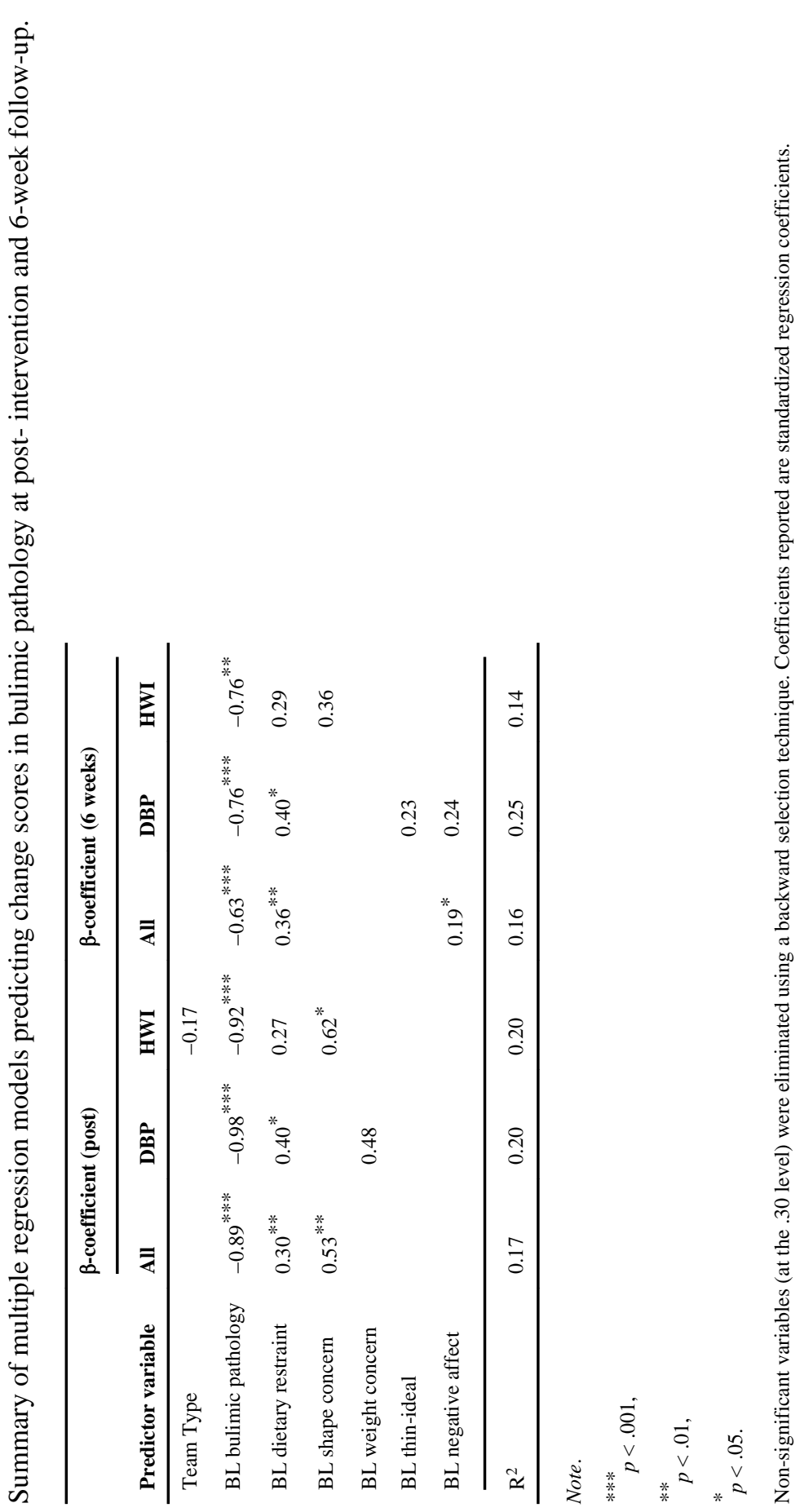

Psychol Sport Exerc. Author manuscript; available in PMC 2015 November 01. 